\title{
On the multi-spin magnon and spike solutions from membranes
}

\author{
P. Bozhilov ${ }^{\star 1}$ and R.C. Rashkov ${ }^{\dagger 2}$ \\ * Institute for Nuclear Research and Nuclear Energy, Bulgarian Academy of Sciences, \\ 1784 Sofia, Bulgaria \\ ${ }^{\dagger}$ Institute for Theoretical Physics, Vienna University of Technology, \\ Wiedner Hauptstr. 8-10, 1040 Vienna, Austria
}

\begin{abstract}
Recently important classes of solitonic string solutions were obtained - giant magnons and single spikes. In previous study we showed the existence of giant magnon-like membrane solutions and studied their properties. In this paper we investigate classical rotating membranes representing analog of a specific class of string spiky solutions. Using the reduction to the Neumann-Rosochatius integrable system we find analog of the string single spike solutions. In contrast to the magnon-like solutions, this case is characterized with finite difference of energy and "winding number" and finite spins as well.
\end{abstract}

\section{Introduction}

Recent advances indicate that integrable structures play an important role in relating string/Mtheory and the gauge theories. One of the conjectures of the correspondence is the direct relation between the spectrum of anomalous dimensions of gauge invariant operators in the the gauge theory and the energy spectrum of the string/M-theory. Therefore, one of the important issues is the dispersion relations in the string/M-theory. The techniques of integrable systems have therefore become useful in studying this correspondence in details.

The strong/weak nature of the duality between string/M-theory and gauge theory makes the proof of the conjectured correspondence difficult. Basically, when the perturbative study is valid on one side of the correspondence, one cannot trust the result from the other, strong coupled theory. There exist however special regimes where one can do reliable calculations on both side of the correspondence. Assuming integrability, in the strong-coupling limit the $S$ matrix can be interpreted as describing the two-body scattering of elementary excitations on the worldvolume. When their worldvolume momenta become large, these excitations can be described as special

\footnotetext{
${ }^{1}$ e-mail: bozhilov@inrne.bas.bg

2e-mail: rash@hep.itp.tuwien.ac.at, on leave from Dept of Physics, Sofia University, Bulgaria.
} 
types of solitonic solutions, or giant magnons, and the interpolating region is described by the dynamics of the so-called near-flat-space regime $[1,2]$. On the gauge theory side, the action of the dilatation operator on single-trace operators is the same as that of a Hamiltonian acting on the states of a certain spin chain [3]. This turns out to be of great advantage because one can diagonalize the matrix of anomalous dimensions by using the algebraic Bethe ansatz technique (see [4] for a nice review on the algebraic Bethe ansatz). In this picture the dynamics involves diffractionless scattering encoded in the S matrix. Proving that the gauge and string/M theories are identical in the planar limit therefore amounts to showing that the underlying physics of both theories is governed by the same two-body scattering matrix.

This approach was developed mainly for checking string/gauge theory duality. It is still not quite well understood in the case of M-theory/gauge theory correspondence due to the complexity of the latter. It is worth however to study particular solutions of the above type for at least three reasons. First, obtaining solutions of stringy type for membranes is interesting on its own and is expected to shed light on some properties of the theory. Secondly, one can gain some insights about how the correspondence should relate the spectrum to the gauge theory operators and identify the latter, and finally it should be possible to reproduce the string result upon dimensional reduction from eleven to ten dimensions.

Recently Hofman and Maldacena [1] were able to map spin chain "magnon" excitations to specific rotating semiclassical string states on $R \times S^{2}$. The relation between energy and angular momentum for the one spin giant magnon found in [1] is:

$$
E-J=\frac{\sqrt{\lambda}}{\pi}\left|\sin \frac{p}{2}\right|
$$

where $p$ is the magnon momentum which on the string side is interpreted as a difference in the angle $\phi$ (see [1] for details). These classical string solutions were further generalized to include magnon bound states $\left([5],[6],[7],[8]\right.$, as well as dynamics on the whole $S^{5}[9]$, [10] and in fact a method to construct classical string solutions describing superposition of arbitrary scattering and bound states was found [11]. The semiclassical quantization of the giant magnon solution was performed in $[12]^{3}$.

A class of classical string solutions with spikes may be of interest for the AdS/CFT correspondence as well. These spiky strings were constructed in the $A d S_{5}$ subspace of $A d S_{5} \times S^{5}$ [26] and it was argued that they correspond to single trace operators with a large number of derivatives. The spiky strings were generalized to include dynamics in the $S^{5}$ part of $A d S_{5} \times S^{5}$ [27] and in [28] closed strings with "kinks" were considered. Some generalizations can be found also in $[29,30]$.

Leaded by the recent developments in string theory, we would like to study the existence of so called single spike solutions $[31,32,33]$ in the membrane case. As it is already known, one can find a consistent reduction of the membrane to the Neumann-Rosochatius integrable system [34] and therefore one can expect that such solutions can be obtained in this case as well.

The paper is organized as follows. In the next section we give short review of the single spike sector in the string case. To analyze the problem we use reduction to Neumann-Rosochatius (NR) integrable system and reproduce some results from [31, 32, 33]. Next we proceed with the analysis of the membrane case. We use the same technique, namely reduction to the NR

\footnotetext{
${ }^{3}$ See also $[13]-[25]$.
} 
system, to obtain the corresponding dispersion relations. We summarize our study in short concluding section.

\section{$2 \quad$ Spiky solutions - string case}

To obtain single spike solutions we will use the reduction to the NR system. This approach is convenient because it will be used in the membrane case and then the comparison and dimensional reduction become transparent. We start with the Polyakov sigma model action supplied with the corresponding Visrasoro constraints.

\section{The Lagrangian}

To write the Lagrangian of the theory we use the following parametrization

$$
x_{a}(\xi)=r_{a}(\xi) e^{i \mu_{a}(\xi)}, \quad\left|x_{a}^{\prime}\right|^{2}=r_{a}^{\prime 2}+r_{a}^{2} \mu_{a}^{\prime 2}, \quad \xi=\alpha \sigma+\beta \tau .
$$

Using parametrization in terms of complex variables as above one can find the Lagrangian

$$
\begin{array}{r}
\mathcal{L}=\sum_{a}\left[\left(\alpha^{2}-\beta^{2}\right) r_{a}^{\prime 2}+\left(\alpha^{2}-\beta^{2}\right) r_{a}^{2}\left(\mu_{a}^{\prime}-\frac{\beta \omega_{a}}{\alpha^{2}-\beta^{2}}\right)^{2}-\frac{\alpha^{2}}{\alpha^{2}-\beta^{2}} \omega_{a}^{2} r_{a}^{2}\right] \\
+\Lambda\left(\sum_{a} r_{a}^{2}-1\right) .
\end{array}
$$

Integrating once the equations of motion for $\mu_{a}$, we get

$$
\mu_{a}^{\prime}=\frac{1}{\alpha^{2}-\beta^{2}}\left(\frac{C_{a}}{r_{a}^{2}}+\beta \omega_{a}\right)
$$

where $C_{a}$ are constants of motion. On other hand for $r_{a}$ we get

$$
\left(\alpha^{2}-\beta^{2}\right) r_{a}^{\prime \prime}-\frac{C_{a}^{2}}{\alpha^{2}-\beta^{2}} \frac{1}{r_{a}^{3}}+\frac{\alpha^{2}}{\alpha^{2}-\beta^{2}} \omega_{a}^{2} r_{a}-\Lambda r_{a}=0
$$

where we used the equations for $\mu(2.4)$.

The equations for $r_{a}$ can be then obtained from the effective Lagrangian

$$
\mathcal{L}=\sum_{a}\left[\left(\alpha^{2}-\beta^{2}\right){r_{a}^{\prime}}^{2}-\frac{C_{a}^{2}}{\alpha^{2}-\beta^{2}} \frac{1}{r_{a}^{2}}-\frac{\alpha^{2}}{\alpha^{2}-\beta^{2}} \omega_{a}^{2} r_{a}^{2}\right]+\Lambda\left(\sum_{a} r_{a}^{2}-1\right)
$$

This few steps were just to make link to string case where the corresponding solutions are already known. One can easily find the corresponding Hamiltonian

$$
\mathcal{H}=\sum_{a}\left[\left(\alpha^{2}-\beta^{2}\right){r_{a}^{\prime}}^{2}+\frac{C_{a}^{2}}{\alpha^{2}-\beta^{2}} \frac{1}{r_{a}^{2}}+\frac{\alpha^{2}}{\alpha^{2}-\beta^{2}} \omega_{a}^{2} r_{a}^{2}\right]
$$




\section{Conformal constraints in NR}

An important part of the analysis are Virasoro constraints

$$
\begin{aligned}
& \sum_{a}\left[\left|\partial_{\tau} X_{a}\right|^{2}+\left|\partial_{\sigma} X_{a}\right|^{2}\right]=\kappa^{2} \\
& \sum_{a}\left[\partial_{\tau} X_{a} \partial_{\sigma} \bar{X}_{a}+\partial_{\tau} \bar{X}_{a} \partial_{\sigma} X_{a}\right]=0
\end{aligned}
$$

where we used

$$
X_{a}=x_{a}(\xi) e^{i \omega_{a} \tau}, \quad \xi=\alpha \sigma+\beta \tau, \quad x_{a}(\xi+2 \pi \alpha)=x_{a}(\xi)
$$

One can use the ansatz (2.2) and the solutions (2.4) to find constraints in terms of the parameters. Namely, one can write the final form of the Virasoro constraints as

$$
\begin{aligned}
& H=\frac{\alpha^{2}+\beta^{2}}{\alpha^{2}-\beta^{2}} \kappa^{2} \\
& \sum_{a} \omega_{a} C_{a}+\beta \kappa^{2}=0
\end{aligned}
$$

The periodicity conditions on $r_{a}$ and $\mu_{a}$ should be imposed and they are as follows

$$
r_{a}(\xi+2 \pi \alpha)=r_{a}(\xi), \quad \mu_{a}(\xi+2 \pi \alpha)=\mu_{a}(\xi)+2 \pi n_{a}, n_{a} \text { - integer }
$$

\section{Two-spin spiky solutions}

What we have to do is to satisfy the constraints $(2.11),(2.12)$ in a way that we get single spike solutions. The two-spin case means that we reduce the system to the $S^{3}$ subspace, or $r_{3}=\mu_{3}=0$ (also $C_{3}=0$ ). In addition, the condition that one of the turning points of the string is at $\theta=\pi / 2$ imposes $C_{2}=0, \beta=-\frac{\omega_{1} C_{1}}{\kappa^{2}}$ (we set $\alpha=1$ and then $|\beta|$ becomes a "group velocity"). The equation for $\kappa$ has two solutions: $\kappa=\omega_{1}$ corresponding to a magnon type solution, and $\kappa=C_{1}$ corresponding to single spike solution.

The single spike choice for $\kappa$ and other parameters is ${ }^{4}$

$$
\kappa=C_{1}, \quad \beta=-\frac{\omega_{1} C_{1}}{\kappa^{2}}=-\frac{\omega_{1}}{C_{1}}
$$

We have to solve the following equation

$$
\frac{d u}{d \xi}=u^{\prime}=2 \frac{\sqrt{\Delta \omega^{2}}}{1-\beta^{2}}(1-u) \sqrt{u-\bar{u}}
$$

where

$$
\begin{aligned}
& u=r_{1}^{2}=\sin ^{2} \theta, \quad \bar{u}=\frac{C_{1}^{2}}{\sqrt{\Delta \omega^{2}}}, \quad \Delta \omega^{2}=\omega_{1}^{2}-\omega_{2}^{2}, \\
& d \xi=\frac{d u}{u^{\prime}}=\frac{\left(1-\beta^{2}\right) d u}{2 \sqrt{\Delta \omega^{2}}(1-u) \sqrt{u-\bar{u}}}=\frac{\left(C_{1}^{2}-\omega_{1}^{2}\right) d u}{2 C_{1}^{2} \sqrt{\Delta \omega^{2}}(1-u) \sqrt{u-\bar{u}}}
\end{aligned}
$$

\footnotetext{
${ }^{4}$ For details see $[31,32,33]$
} 
The conserved quantities are

$$
\begin{aligned}
\hat{\mu}_{1} & =\frac{C_{1}}{\left(1-\beta^{2}\right)} \int \frac{d \xi}{u}+\frac{\beta \omega_{1}}{\left(1-\beta^{2}\right)} \int d \xi \\
E & =\kappa T \int d \xi \\
J_{1} & =\frac{C_{1} \beta}{\left(1-\beta^{2}\right)} \int d \xi+\frac{\omega_{1}}{\left(1-\beta^{2}\right)} T \int u d \xi \\
J_{2} & =\frac{\omega_{2}}{\left(1-\beta^{2}\right)} T \int(1-u) d \xi
\end{aligned}
$$

where $\hat{\mu}_{1}$ is the angular extension of the string

$$
\hat{\mu}_{1}=\int \mu_{1}^{\prime} d \xi
$$

Let us consider the following difference

$$
E-T \hat{\mu}_{1}=\frac{2 C_{1} T}{\sqrt{\Delta \omega^{2}}} \arccos \sqrt{\bar{u}}=\frac{\sqrt{\lambda}}{\pi} \bar{\theta}
$$

where

$$
\bar{\theta}=\frac{\pi}{2}-\theta_{0}
$$

Unlike in the case of giant magnons, this difference is finite (and the energy is still large). This means that the string has one spike, but is wounded many times along the equator of $S^{5}$ so that the difference between the energy and winding number $T \hat{\mu}_{1}$ is finite.

For the spin $J_{1}$ we get

$$
J_{1}=\frac{2 T \omega_{1}}{\sqrt{\Delta \omega^{2}}} \cos \theta_{0}=\frac{2 T \omega_{1}}{\sqrt{\Delta \omega^{2}}} \sin \bar{\theta}
$$

and analogously for $J_{2}$ we find

$$
J_{2}=-\frac{2 T \omega_{2}}{\sqrt{\Delta \omega^{2}}} \cos \theta_{0}=-\frac{2 T \omega_{2}}{\sqrt{\Delta \omega^{2}}} \sin \bar{\theta}
$$

Defining $\sin \gamma$ as in [31], equation (6.23) (where it is actually wrong because $\omega_{1}>\omega_{2}$ )

$$
\sin \gamma=\frac{\omega_{2}}{\omega_{1}}, \quad \sin \theta_{0}=\frac{C_{1}}{\sqrt{\Delta \omega^{2}}}
$$

we one can write $J_{i}$ as

$$
\begin{aligned}
& J_{1}=2 T \frac{1}{\sin \gamma} \sin \bar{\theta} \\
& J_{2}=-2 T \frac{\sin \gamma}{\cos \gamma} \sin \bar{\theta}
\end{aligned}
$$

Note that both $J_{i}$ are finite. 
Eliminating the auxiliary parameter $\gamma$ one can obtain the following dispersion relations

$$
\begin{aligned}
& E-T \hat{\mu}_{1}=\frac{\sqrt{\lambda}}{\pi} \bar{\theta} \\
& J_{1}=\sqrt{J_{2}^{2}+\frac{\lambda}{\pi^{2}} \sin ^{2} \bar{\theta}} .
\end{aligned}
$$

We see that in the limit $J_{2}=0$ we reproduce the one-spin case. One can define the anomalous dimensions in this case $\left(J_{2}=0\right.$ and $\left.J_{1}=J\right)$ as in the magnon considerations but subtracting the winding number $T \hat{\mu}_{1}$ to get "renormalized" result

$$
\Delta=\left(E-T \hat{\mu}_{1}\right)-J=\frac{\sqrt{\lambda}}{\pi}(\bar{\theta}-\sin \bar{\theta}),
$$

which reproduces the result of $[31,32,33]$.

\section{$3 \quad$ Membranes on $A d S_{4} \times S^{7}$}

Turning to the membrane case, let us first write down the gauge fixed membrane action and constraints in diagonal worldvolume gauge, we are going to work with:

$$
\begin{aligned}
& S_{M}=\int d^{3} \xi \mathcal{L}_{M}=\int d^{3} \xi\left\{\frac{1}{4 \lambda^{0}}\left[G_{00}-\left(2 \lambda^{0} T_{2}\right)^{2} \operatorname{det} G_{i j}\right]+T_{2} C_{012}\right\} \\
& G_{00}+\left(2 \lambda^{0} T_{2}\right)^{2} \operatorname{det} G_{i j}=0 \\
& G_{0 i}=0 .
\end{aligned}
$$

They coincide with the frequently used gauge fixed Polyakov type action and constraints after the identification $2 \lambda^{0} T_{2}=L=$ const, where $\lambda^{0}$ is Lagrange multiplier and $T_{2}$ is the membrane tension. In (3.1)-(3.3), the fields induced on the membrane worldvolume $G_{m n}$ and $C_{012}$ are given by

$$
\begin{aligned}
& G_{m n}=g_{M N} \partial_{m} X^{M} \partial_{n} X^{N}, \quad C_{012}=c_{M N P} \partial_{0} X^{M} \partial_{1} X^{N} \partial_{2} X^{P} \\
& \partial_{m}=\partial / \partial \xi^{m}, \quad m=(0, i)=(0,1,2), \\
& \left(\xi^{0}, \xi^{1}, \xi^{2}\right)=\left(\tau, \sigma_{1}, \sigma_{2}\right), \quad M=(0,1, \ldots, 10),
\end{aligned}
$$

where $g_{M N}$ and $c_{M N P}$ are the components of the target space metric and 3-form gauge field respectively.

Searching for membrane solutions in $A d S_{4} \times S^{7}$ analogous to string ones on $A d S_{5} \times S^{5}$, we should first eliminate the membrane interaction with the background 3-form field on $A d S_{4}$. To make our choice, let us write down the background. It can be parameterized as follows

$$
\begin{aligned}
& d s^{2}=\left(2 l_{p} \mathcal{R}\right)^{2}\left[-\cosh ^{2} \rho d t^{2}+d \rho^{2}+\sinh ^{2} \rho\left(d \alpha^{2}+\sin ^{2} \alpha d \beta^{2}\right)+4 d \Omega_{7}^{2}\right], \\
& c_{(3)}=\left(2 l_{p} \mathcal{R}\right)^{3} \sinh ^{3} \rho \sin \alpha d t \wedge d \alpha \wedge d \beta .
\end{aligned}
$$

Since we want the membrane to have nonzero conserved energy and spin on $A d S$, the choice for which the interaction with the $c_{(3)}$ field disappears is ${ }^{5}$ :

$$
\alpha=\alpha_{0}=\text { const. }
$$

\footnotetext{
${ }^{5}$ Of course, we can fix the angle $\beta$ instead of $\alpha$. We choose to fix $\alpha$ because $\beta$ is one of the isometry coordinates in the initial $A d S_{4}$ space.
} 
The metric of the corresponding subspace of $A d S_{4}$ is

$$
\begin{aligned}
& d s_{\text {sub }}^{2}=\left(2 l_{p} \mathcal{R}\right)^{2}\left(-\cosh ^{2} \rho d t^{2}+d \rho^{2}+\sinh ^{2} \rho \sin ^{2} \alpha_{0} d \beta^{2}\right)= \\
& \left(2 l_{p} \mathcal{R}\right)^{2}\left[-\cosh ^{2} \rho d t^{2}+d \rho^{2}+\sinh ^{2} \rho d\left(\beta \sin \alpha_{0}\right)^{2}\right] .
\end{aligned}
$$

Hence, the appropriate membrane embedding into (3.5) and $S^{7}$, analogous to the string embedding in $A d S_{5} \times S^{5}$, is

$$
\begin{array}{ll}
Z_{\mu}=2 l_{p} \mathcal{R r}_{\mu}\left(\xi^{m}\right) e^{i \phi_{\mu}\left(\xi^{m}\right)}, & \mu=(0,1), \quad \phi_{\mu}=\left(\phi_{0}, \phi_{1}\right)=\left(t, \beta \sin \alpha_{0}\right), \\
& \eta^{\mu \nu} \mathrm{r}_{\mu} \mathrm{r}_{\nu}+1=0, \quad \eta^{\mu \nu}=(-1,1), \\
W_{a}=4 l_{p} \mathcal{R} r_{a}\left(\xi^{m}\right) e^{i \varphi_{a}\left(\xi^{m}\right)}, \quad a=(1,2,3,4), \quad \delta_{a b} r_{a} r_{b}-1=0 .
\end{array}
$$

For this embedding, the induced metric is given by

$$
\begin{aligned}
& G_{m n}=\eta^{\mu \nu} \partial_{(m} Z_{\mu} \partial_{n)} \bar{Z}_{\nu}+\delta_{a b} \partial_{(m} W_{a} \partial_{n)} \bar{W}_{b}= \\
& \left(2 l_{p} \mathcal{R}\right)^{2}\left[\sum_{\mu, \nu=0}^{1} \eta^{\mu \nu}\left(\partial_{m} \mathrm{r}_{\mu} \partial_{n} \mathrm{r}_{\nu}+\mathrm{r}_{\mu}^{2} \partial_{m} \phi_{\mu} \partial_{n} \phi_{\nu}\right)+4 \sum_{a=1}^{4}\left(\partial_{m} r_{a} \partial_{n} r_{a}+r_{a}^{2} \partial_{m} \varphi_{a} \partial_{n} \varphi_{a}\right)\right] .
\end{aligned}
$$

We will use the expression (3.7) for $G_{m n}$ in (3.1), (3.2) and (3.3). Correspondingly, the membrane Lagrangian becomes

$$
\mathcal{L}=\mathcal{L}_{M}+\Lambda_{A}\left(\eta^{\mu \nu} \mathrm{r}_{\mu} \mathrm{r}_{\nu}+1\right)+\Lambda_{S}\left(\delta_{a b} r_{a} r_{b}-1\right),
$$

where $\Lambda_{A}$ and $\Lambda_{S}$ are Lagrange multipliers.

In this paper, we are interested in the following particular case of the membrane embedding (3.6)

$$
Z_{0}=2 l_{p} \mathcal{R} e^{i \kappa \tau}, \quad Z_{1}=0
$$

which implies

$$
\mathrm{r}_{0}=1, \quad \mathrm{r}_{1}=0, \quad \phi_{0}=t=\kappa \tau .
$$

For this ansatz, the metric induced on the membrane worldvolume simplifies to

$$
G_{m n}=\left(4 l_{p} \mathcal{R}\right)^{2}\left[\sum_{a=1}^{4}\left(\partial_{m} r_{a} \partial_{n} r_{a}+r_{a}^{2} \partial_{m} \varphi_{a} \partial_{n} \varphi_{a}\right)-\delta_{m}^{0} \delta_{n}^{0}(\kappa / 2)^{2}\right],
$$

and the membrane Lagrangian is given by

$$
\mathcal{L}=\mathcal{L}_{M}+\Lambda_{S}\left(\sum_{a=1}^{4} r_{a}^{2}-1\right)
$$

The known most general membrane embedding in $A d S_{4} \times S^{7}$ leading to the Neumann Rosochatius integrable system is [34]

$$
\begin{aligned}
& Z_{0}=2 l_{p} \mathcal{R} e^{i \kappa \tau}, \quad Z_{1}=0, \quad W_{a}=4 l_{p} \mathcal{R} r_{a}(\xi, \eta) e^{i\left[\omega_{a} \tau+g_{a}(\xi, \eta)\right]} \\
& \xi=\alpha \sigma_{1}+\beta \tau, \quad \eta=\gamma \sigma_{2}+\delta \tau, \quad \alpha, \beta, \gamma, \delta=\text { constants }
\end{aligned}
$$


for

$$
\begin{aligned}
& r_{1}=r_{1}(\xi), \quad r_{2}=r_{2}(\xi), \quad \omega_{3}= \pm \omega_{4}=\omega \\
& r_{3}=r_{3}(\eta)=a \sin (b \eta+c), \quad r_{4}=r_{4}(\eta)=a \cos (b \eta+c), \quad a<1, \\
& g_{1}=g_{1}(\xi), \quad g_{2}=g_{2}(\xi), \quad a, b, c, g_{3}, g_{4}=\text { constants }, \quad \delta=0 .
\end{aligned}
$$

The above ansatz reduces the membrane Lagrangian $\mathcal{L}$ in $(3.11)$ to

$$
\begin{aligned}
\mathcal{L}_{*}^{M} & =-\frac{\left(2 l_{p} \mathcal{R}\right)^{2}}{\lambda^{0}}\left\{\sum _ { a = 1 } ^ { 2 } \left[\left(\tilde{A}^{2}-\beta^{2}\right)\left(\partial_{\xi} r_{a}\right)^{2}+\left(\tilde{A}^{2}-\beta^{2}\right) r_{a}^{2}\left(\partial_{\xi} g_{a}-\frac{\beta \omega_{a}}{\tilde{A}^{2}-\beta^{2}}\right)^{2}\right.\right. \\
& \left.\left.-\frac{\tilde{A}^{2}}{\tilde{A}^{2}-\beta^{2}} \omega_{a}^{2} r_{a}^{2}\right]+(\kappa / 2)^{2}-(a \omega)^{2}\right\}+\Lambda_{S}\left[\sum_{a=1}^{2} r_{a}^{2}-\left(1-a^{2}\right)\right],
\end{aligned}
$$

where

$$
\tilde{A}^{2} \equiv\left(8 \lambda^{0} T_{2} l_{p} \mathcal{R} a b \alpha \gamma\right)^{2}
$$

As shown in [34], $\mathcal{L}_{*}^{M}$ corresponds to the following Neumann-Rosochatius type Lagrangian

$$
\begin{aligned}
\mathcal{L}_{N R}^{M} & =\frac{\left(2 l_{p} \mathcal{R}\right)^{2}}{\lambda^{0}} \sum_{a=1}^{2}\left[\left(\tilde{A}^{2}-\beta^{2}\right)\left(\partial_{\xi} r_{a}\right)^{2}-\frac{C_{a}^{2}}{\left(\tilde{A}^{2}-\beta^{2}\right) r_{a}^{2}}-\frac{\tilde{A}^{2}}{\tilde{A}^{2}-\beta^{2}} \omega_{a}^{2} r_{a}^{2}\right] \\
& +\Lambda_{S}\left[\sum_{a=1}^{2} r_{a}^{2}-\left(1-a^{2}\right)\right], \quad C_{a}=\text { constants. }
\end{aligned}
$$

For the present case, the constraint $G_{02}=0$ in (3.3) is satisfied identically, due to $\delta=0$. The other two constraints in (3.2) and (3.3) can be written in the form

$$
\begin{aligned}
H \sim & \sum_{a=1}^{2}\left[\left(\tilde{A}^{2}-\beta^{2}\right)\left(\partial_{\xi} r_{a}\right)^{2}+\frac{C_{a}^{2}}{\left(\tilde{A}^{2}-\beta^{2}\right) r_{a}^{2}}+\frac{\tilde{A}^{2}}{\tilde{A}^{2}-\beta^{2}} \omega_{a}^{2} r_{a}^{2}\right] \\
& =\frac{\tilde{A}^{2}+\beta^{2}}{\tilde{A}^{2}-\beta^{2}}\left[(\kappa / 2)^{2}-(a \omega)^{2}\right] \\
& \sum_{a=1}^{2} \omega_{a} C_{a}+\beta\left[(\kappa / 2)^{2}-(a \omega)^{2}\right]=0
\end{aligned}
$$

where $r_{a}$ must satisfy the condition

$$
\sum_{a=1}^{2} r_{a}^{2}-\left(1-a^{2}\right)=0 .
$$

Parameterizing the circle (3.17) by

$$
r_{1}=\left(1-a^{2}\right)^{1 / 2} \cos \psi, \quad r_{2}=\left(1-a^{2}\right)^{1 / 2} \sin \psi,
$$

one obtains that (3.17) is satisfied identically and (3.15) reduces to (prime is used for $d / d \xi$ )

$$
\begin{aligned}
\psi^{\prime 2}= & \frac{1}{\left(\tilde{A}^{2}-\beta^{2}\right)^{2}\left(1-a^{2}\right)}\left\{\left(\tilde{A}^{2}+\beta^{2}\right)\left[(\kappa / 2)^{2}-(a \omega)^{2}\right]\right. \\
& \left.-\frac{1}{1-a^{2}}\left(\frac{C_{1}^{2}}{\cos ^{2} \psi}+\frac{C_{2}^{2}}{\sin ^{2} \psi}\right)-\tilde{A}^{2}\left(1-a^{2}\right)\left(\omega_{1}^{2} \cos ^{2} \psi+\omega_{2}^{2} \sin ^{2} \psi\right)\right\} .
\end{aligned}
$$




\section{One-spin solutions}

If we set $C_{1}=\omega_{1}=0$, from (3.16) and (3.18) we get

$$
\psi^{\prime}= \pm \frac{\tilde{A} \omega_{2}}{\left(\tilde{A}^{2}-\beta^{2}\right) \sin \psi} \sqrt{\left(\sin ^{2} \psi_{0}-\sin ^{2} \psi\right)\left(\sin ^{2} \psi-\sin ^{2} \psi_{1}\right)}
$$

where

$$
\sin ^{2} \psi_{0}=\frac{(\kappa / 2)^{2}-(a \omega)^{2}}{\omega_{2}^{2}\left(1-a^{2}\right)}, \quad \sin ^{2} \psi_{1}=\frac{\beta^{2}}{\tilde{A}^{2}} \sin ^{2} \psi_{0}
$$

The equation (3.19) gives the same spiky and giant magnon solutions as (5.11) of [31].

\section{Two-spin solutions}

Following [33], for obtaining two-spin, spiky and giant magnon solutions, we set $C_{1}=0$ in (3.18) and taking into account (3.16), we receive

$$
\begin{aligned}
\psi^{\prime 2}= & \frac{\tilde{A}^{2}\left(\omega_{2}^{2}-\omega_{1}^{2}\right)}{\left(\tilde{A}^{2}-\beta^{2}\right)^{2} \sin ^{2} \psi}\left\{\frac{(\kappa / 2)^{2}-(a \omega)^{2}}{\left(1-a^{2}\right)\left(\omega_{2}^{2}-\omega_{1}^{2}\right)}\left[1+\frac{\beta^{2}}{\tilde{A}^{2}}-\frac{\left(1-a^{2}\right) \omega_{1}^{2}}{(\kappa / 2)^{2}-(a \omega)^{2}}\right] \sin ^{2} \psi\right. \\
& \left.-\frac{\beta^{2}\left[(\kappa / 2)^{2}-(a \omega)^{2}\right]^{2}}{\tilde{A}^{2}\left(1-a^{2}\right)^{2} \omega_{2}^{2}\left(\omega_{2}^{2}-\omega_{1}^{2}\right)}-\sin ^{4} \psi\right\} .
\end{aligned}
$$

The solutions we are searching for, correspond to two particular choices of the parameters in the above expression for $\psi^{\prime 2}$.

The first choice is to set in (3.20)

$$
(\kappa / 2)^{2}-(a \omega)^{2}=\left(1-a^{2}\right) \omega_{2}^{2} .
$$

This results in

$$
\psi^{\prime}=\frac{\tilde{A} \sqrt{\omega_{2}^{2}-\omega_{1}^{2}}}{\tilde{A}^{2}-\beta^{2}} \frac{\cos \psi}{\sin \psi} \sqrt{\sin ^{2} \psi-\sin ^{2} \psi_{0}},
$$

where

$$
\sin ^{2} \psi_{0}=\frac{\beta^{2} \omega_{2}^{2}}{\tilde{A}^{2}\left(\omega_{2}^{2}-\omega_{1}^{2}\right)}
$$

Comparing (3.21) with (3.23) in [33], we see that this equation for $\psi$ leads to giant magnon solution on $S^{3}$.

The second appropriate choice is

$$
(\kappa / 2)^{2}-(a \omega)^{2}=\frac{\tilde{A}^{2}\left(1-a^{2}\right) \omega_{2}^{2}}{\beta^{2}} .
$$

Then, (3.20) reduces to

$$
\psi^{\prime}=\frac{\tilde{A} \sqrt{\omega_{2}^{2}-\omega_{1}^{2}}}{\tilde{A}^{2}-\beta^{2}} \frac{\cos \psi}{\sin \psi} \sqrt{\sin ^{2} \psi-\sin ^{2} \psi_{1}},
$$


where

$$
\sin ^{2} \psi_{1}=\frac{\tilde{A}^{2} \omega_{2}^{2}}{\beta^{2}\left(\omega_{2}^{2}-\omega_{1}^{2}\right)}
$$

Comparing (3.22) with (3.28) in [33], we see that this equation for $\psi$ leads to single spike solution on $S^{3}$, first obtained in [31] from the Nambu-Goto string action.

Let us finally note that both equations (3.21) and (3.22) are of the type

$$
\frac{d u}{d \xi}=u^{\prime}=2 \tilde{K}(1-u) \sqrt{u-\bar{u}}, \quad \tilde{K}=\frac{\tilde{A} \sqrt{\omega_{2}^{2}-\omega_{1}^{2}}}{\tilde{A}^{2}-\beta^{2}}, \quad u=\sin ^{2} \psi,
$$

corresponding to different values of the parameter $\bar{u}$ :

$$
\bar{u}=\sin ^{2} \psi_{0}, \quad \text { or } \quad \bar{u}=\sin ^{2} \psi_{1} \text {. }
$$

\section{Conserved quantities}

The energy $E$ and the angular momenta $J_{a}$ can be computed by using the equalities

$$
E=-\int d^{2} \sigma \frac{\partial \mathcal{L}}{\partial\left(\partial_{0} t\right)}, \quad J_{a}=\int d^{2} \sigma \frac{\partial \mathcal{L}}{\partial\left(\partial_{0} \varphi_{a}\right)}, \quad a=1,2,3,4
$$

For the embedding (3.12), (3.13), they are given by

$$
\begin{aligned}
E & =\frac{\pi\left(2 l_{p} \mathcal{R}\right)^{2} \kappa}{\lambda^{0} \alpha} \int d \xi \\
J_{a} & =\frac{\pi\left(4 l_{p} \mathcal{R}\right)^{2}}{\lambda^{0} \alpha\left(\tilde{A}^{2}-\beta^{2}\right)} \int d \xi\left(\beta C_{a}+\tilde{A}^{2} \omega_{a} r_{a}^{2}\right), \quad a=1,2 .
\end{aligned}
$$

In order to reproduce the string case, we set $\omega=0$, which leads to $J_{3}=J_{4}=0$.

On the solution for $\xi(u), E$ and $J_{a}$ take the form

$$
\begin{aligned}
& E=\frac{\pi\left(2 l_{p} \mathcal{R}\right)^{2} \kappa}{\lambda^{0} \alpha \tilde{K}} \int_{\bar{u}}^{1} \frac{d u}{(1-u) \sqrt{u-\bar{u}}}, \\
& J_{1}=\frac{\pi\left(4 l_{p} \mathcal{R}\right)^{2} \tilde{A}^{2}\left(1-a^{2}\right) \omega_{1}}{\lambda^{0} \alpha \tilde{K}\left(\tilde{A}^{2}-\beta^{2}\right)} \int_{\bar{u}}^{1} \frac{d u}{\sqrt{u-\bar{u}}}, \\
& J_{2}=\frac{\pi\left(4 l_{p} \mathcal{R}\right)^{2}}{\lambda^{0} \alpha \tilde{K}\left(\tilde{A}^{2}-\beta^{2}\right)}\left\{\left[\tilde{A}^{2}\left(1-a^{2}\right) \omega_{2}-\frac{\beta^{2}}{\omega_{2}}(\kappa / 2)^{2}\right] \int_{\bar{u}}^{1} \frac{d u}{(1-u) \sqrt{u-\bar{u}}}\right. \\
& \left.-\tilde{A}^{2}\left(1-a^{2}\right) \omega_{2} \int_{\bar{u}}^{1} \frac{d u}{\sqrt{u-\bar{u}}}\right\} .
\end{aligned}
$$

From (3.23), for the giant magnon case, one obtains the energy-charge relation

$$
E-\frac{J_{2}}{2 \sqrt{1-a^{2}}}=\sqrt{\left(\frac{J_{1}}{2 \sqrt{1-a^{2}}}\right)^{2}+\left[2^{7} \pi T_{2}\left(l_{p} \mathcal{R}\right)^{3} a \sqrt{1-a^{2}} b \gamma\right]^{2} \cos ^{2} \psi_{0}}
$$


The origin of the denominators $2 \sqrt{1-a^{2}}$ in the above equality can be explained as follows. The factor of 2 is due to the different radii of $A d S_{4}$ and $S^{7}$, while the radii of $A d S_{5}$ and $S^{5}$ in type IIB string background $A d S_{5} \times S^{5}$ are equal. $\sqrt{1-a^{2}}$ arises because the NR system obtained from membrane is restricted to lie on a circle with radius $\sqrt{1-a^{2}}$, whereas in the string case this radius equals one.

For the single spike solution using (3.23) again, one arrives at

$$
J_{2}=\sqrt{J_{1}^{2}+\left[2^{8} \pi T_{2}\left(l_{p} \mathcal{R}\right)^{3} a\left(1-a^{2}\right) b \gamma\right]^{2} \cos ^{2} \psi_{1}}
$$

Now following [10], we introduce the angular extension of the membrane as

$$
\hat{g}_{2}=\int g_{2}^{\prime} d \xi=\frac{1}{\tilde{A}^{2}-\beta^{2}} \int\left(\frac{C_{2}}{r_{2}^{2}}+\beta \omega_{2}\right) d \xi
$$

and compute the difference $E-\tilde{T} \hat{g}_{2}$. It turns out it is finite for

$$
\tilde{T}=2^{6} \pi T_{2}\left(l_{p} \mathcal{R}\right)^{3} a \sqrt{1-a^{2}} b \gamma
$$

and is given by

$$
E-\tilde{T} \hat{g}_{2}=2 \tilde{T}\left(\frac{\pi}{2}-\psi_{1}\right)
$$

Comparing (3.24), (3.25) with (2.25), (2.24), we see that the single spike dispersion relations obtained from membrane and from string are of the same type.

\section{Conclusions}

It is well known that particular solitonic solutions in string/M theory are important not only on their own but also because of their relations to the gauge theories and for integrability issues as well. The development in obtaining such solutions in string theory inspires the interest to analogous investigations in $\mathrm{M}$ theory. In this short letter we investigated the issue of existence of spike-like solutions in membrane case. These solutions extend the class of previously found "giant magnon" solutions at the large energy scale. Concretely, these issues are interesting for the following reasons. The results for type IIB strings should be reproduced taking an appropriate dimensional reduction. Then the question whether the known string solutions have analog in eleven dimensional space-time appears. The answer to this question for the giant magnon string solutions is positive [35]. It is natural to extend the analysis to the case of so called "single spike" solutions.

First we presented a short review of the string case and demonstrated how one can find solutions of this type using the reduction to the Neumann-Rosochatius integrable system. In the next Section we turn to the membrane case. The analysis shows that there is an analog of this class solutions with the same properties. To show that, we used again the possibility to reduce the theory to a Neumann-Rosochatius integrable system. The solutions are obtained by taking an appropriate limit and the dispersion relations are found to be

$$
\begin{aligned}
& \sqrt{1-a^{2}} E-\frac{J_{2}}{2}=\sqrt{\left(\frac{J_{1}}{2}\right)^{2}+\frac{\tilde{\lambda}}{\pi^{2}} \sin ^{2} \frac{p}{2}}, \\
& \tilde{\lambda}=\left[2^{7} \pi^{2} T_{2}\left(l_{p} \mathcal{R}\right)^{3} a\left(1-a^{2}\right) b \gamma\right]^{2}, \quad \frac{p}{2}=\frac{\pi}{2}-\psi_{0},
\end{aligned}
$$


for the giant magnon solution, and

$$
\begin{aligned}
& E-\tilde{T} \hat{g}_{2}=\frac{\sqrt{\tilde{\lambda}}}{\pi} \bar{\psi}, \\
& \frac{J_{2}}{2}=\sqrt{\left(\frac{J_{1}}{2}\right)^{2}+\frac{\tilde{\lambda}}{\pi^{2}} \sin ^{2} \bar{\psi}} \\
& \tilde{T}=\frac{\sqrt{\tilde{\lambda}}}{2 \pi}, \quad \bar{\psi}=\frac{\pi}{2}-\psi_{1},
\end{aligned}
$$

for the single spike solution, in full analogy with the string case.

Although the membrane/gauge theory correspondence is not well developed, we hope that these results will shed light on the duality. The main obstacle is the lack of clear principle and mechanism to identify the gauge theory operators. Collecting evidences and analogies with strings will show up hopefully such a principle. There are hopes that one can find an integrable spin chain which governs this correspondence at least in the sectors that are known to be integrable but still a lot of work is ahead.

\section{Acknowledgements}

This work was supported in part by Bulgarian NSF grants $F-1412 / 04$ and $V U-F-201 / 06$.

R.R. acknowledges a Guest Professorship and warm hospitality at the Institute for Theoretical Physics, Vienna University of Technology. Many thanks to Max Kreuzer and his group for fruitful and stimulating atmosphere. The work of R.R is partially supported by Austrian Research Fund FWF grant \# P19051-N16.

\section{References}

[1] Diego M. Hofman, Juan Maldacena, Giant Magnons, J. Phys. A 39 (2006) 13095-13118 [arXiv:hep-th/0604135v2].

[2] Juan Maldacena, Ian Swanson, Connecting giant magnons to the pp-wave: An interpolating limit of $A d S_{5} \times S^{5}$, arXiv:hep-th/0612079v4.

[3] J. A. Minahan and K. Zarembo, The Bethe-ansatz for $\mathcal{N}=4$ super Yang-Mills, JHEP 0303 (2003) 013, [arXiv:hep-th/0212208v3].

[4] L. D. Faddeev, How Algebraic Bethe Ansatz works for integrable model, arXiv:hepth/9605187v1.

[5] Nick Dorey, Magnon bound states and the AdS/CFT correspondence, J. Phys. A 39 (2006) 13119-13128, [arXiv:hep-th/0604175v2].

[6] Heng-Yu Chen, Nick Dorey, Keisuke Okamura, Dyonic Giant Magnons, JHEP 0609 (2006) 024, [arXiv:hep-th/0605155v2].

[7] Gleb Arutyunov, Sergey Frolov, Marija Zamaklar, Finite-size Effects from Giant Magnons, doi:10.1016/j.nuclphysb.2006.12.026 [arXiv:hep-th/0606126v2]. 
[8] J.A. Minahan, A. Tirziu, A.A. Tseytlin, Infinite spin limit of semiclassical string states, JHEP 0608 (2006) 049, [arXiv:hep-th/0606145v2].

[9] Marcus Spradlin, Anastasia Volovich, Dressing the Giant Magnon, JHEP 0610 (2006) 012 , [arXiv:hep-th/0607009v3].

[10] M. Kruczenski, J. Russo, A.A. Tseytlin, Spiky strings and giant magnons on $S^{5}$, JHEP 0610 (2006) 002 [arXiv:hep-th/0607044v3].

[11] Chrysostomos Kalousios, Marcus Spradlin, Anastasia Volovich, Dressing the Giant Magnon II, arXiv:hep-th/0611033v1.

[12] Georgios Papathanasiou, Marcus Spradlin, Semiclassical Quantization of the Giant Magnon, arXiv:0704.2389v2 [hep-th].

[13] Wung-Hong Huang, Giant Magnons under NS-NS and Melvin Fields, JHEP 0612 (2006) 040, [arXiv:hep-th/0607161v4].

[14] Keisuke Okamura, Ryo Suzuki, A Perspective on Classical Strings from Complex SineGordon Solitons, Phys. Rev. D 75 (2007) 046001 [arXiv:hep-th/0609026v3].

[15] Shinji Hirano, Fat Magnon, arXiv:hep-th/0610027v4.

[16] Shijong Ryang, Three-Spin Giant Magnons in AdS $\times$ $S^{5}$, JHEP 0612 (2006) 043 [arXiv:hep-th/0610037v1].

[17] Heng-Yu Chen, Nick Dorey, Keisuke Okamura, The Asymptotic Spectrum of the N=4 Super Yang-Mills Spin Chain, arXiv:hep-th/0610295v1.

[18] P. Bozhilov, A note on two-spin magnon-like energy-charge relations from M-theory viewpoint, arXiv:hep-th/0612175v2.

[19] J. A. Minahan, Zero modes for the giant magnon, JHEP 0702 (2007) 048 [arXiv:hepth/0701005v3].

[20] Davide Astolfi, Valentina Forini, Gianluca Grignani, Gordon W. Semenoff, Gauge invariant finite size spectrum of the giant magnon, arXiv:hep-th/0702043v3.

[21] Benoit Vicedo, Giant Magnons and Singular Curves, arXiv:hep-th/0703180v1.

[22] J. Kluson, Rashmi R. Nayak, Kamal L. Panigrahi, Giant Magnon in NS5-brane Background, arXiv:hep-th/0703244v2.

[23] C. A. S. Young, q-Deformed Supersymmetry and Dynamic Magnon Representations, arXiv:0704.2069v2 [hep-th].

[24] Heng-Yu Chen, Nick Dorey, Rui F. Lima Matos, Quantum Scattering of Giant Magnons, arXiv:0707.0668v1 [hep-th].

[25] David Berenstein, Samuel E. Vazquez, Giant magnon bound states from strongly coupled $N=4 S Y M$, arXiv:0707.4669v1 [hep-th]. 
[26] M. Kruczenski, Spiky strings and single trace operators in gauge theories, JHEP 0508 (2005) 014 [arXiv:hep-th/0410226v2].

[27] S. Ryang, Wound and Rotating Strings in $A d S_{5} \times S^{5}$, JHEP 0508 (2005) 047 [arXiv:hepth/0503239v1].

[28] T. McLoughlin and X. Wu, Kinky strings in $A d S_{5} \times S^{5}$, JHEP 0608, 063 (2006) [arXiv:hepth/0604193v2].

[29] Chong-Sun Chu, George Georgiou, Valentin V. Khoze, Magnons, Classical Strings and beta-Deformations, JHEP 0611 (2006) 093, [arXiv:hep-th/0606220v2].

[30] N.P. Bobev, R.C. Rashkov, Multispin Giant Magnons, Phys. Rev. D 74 (2006) 046011, [arXiv:hep-th/0607018v3].

[31] Riei Ishizeki, Martin Kruczenski, Single spike solutions for strings on S2 and S3, arXiv:0705.2429v1 [hep-th].

[32] A. E. Mosaffa, B. Safarzadeh, Dual Spikes; New Spiky String Solutions, arXiv:0705.3131v2 [hep-th].

[33] N.P. Bobev, R.C. Rashkov, Spiky Strings, Giant Magnons and beta - deformations, arXiv:0706.0442v2 [hep-th].

[34] P. Bozhilov, Neumann and Neumann-Rosochatius integrable systems from membranes on $A d S_{4} \times S^{7}$, arXiv:0704.3082v3 [hep-th].

[35] P. Bozhilov, R.C. Rashkov, Magnon-like dispersion relation from M-theory, Nucl. Phys. B 768 [PM] (2007) 193-208 [arXiv:hep-th/0607116v3]. 